IONARA DINIZ EVANGELISTA

SAntos Barcelos ${ }^{1}$

RODOLPHO CRUZ VIEIRA ${ }^{1}$

ELISA MELO FERREIRA

Maria Cristina Picinato

Medeiros de Araújo ${ }^{2}$

Wellington de Paula Martins ${ }^{3}$

RUI Alberto FerRIAN ${ }^{4}$

Paula Andrea de Albuguerque

SALLES NAVARRO 5

\section{Anomalias meióticas de oócitos de pacientes com endometriose submetidas à estimulação ovariana}

\author{
Meiotic abnormalities of oocytes from patients with endometriosis \\ submitted to ovarian stimulation
}

Palavras-chave

Endometriose

Oócitos/crescimento \& desenvolvimento

Oócitos/ ultraestrutura

Fertilização in vitro

Meiose

Técnicas reprodutivas assistidas

Keywords

Endometriosis

Oocytes/growth \& development

Oocytes/ultrastructure

Fertilization in vitro

Meiosis

Reproductive techniques, assisted

Correspondência:

Paula Andrea de Albuquerque Salles Novarro Setor de Reprodução Humana, Departamento de Ginecologia e Obstetrícia da Faculdade de Medicina de Ribeirão Preto do

Universidade de São Paulo

Avenida Bandeirantes, 3.900 - Monte Alegre CEP 14049-900 - Ribeir̃o Preto/SP

Fone: (16) 3602-2821/Fax: 3602-2810 E-mail: pnavarro@fmrp.usp.br

Recebido

$10 / 7 / 08$

Aceito com modificacōos

$7 / 8 / 08$

\section{Resumo}

OBJETIVO: avaliar o fuso meiótico e a distribuição cromossômica de oócitos maturados in vitro, obtidos de ciclos estimulados de mulheres inférteis com endometriose e fatores masculino e/ou tubário de infertilidade (Grupo Controle), comparando as taxas de maturação in vitro (MIV) entre os dois grupos avaliados. MÉTODOS: quatorze pacientes com endometriose e oito com fator tubário ou masculino, submetidas à estimulação ovariana para injeção intracitoplasmática de espermatozóide, foram selecionadas, prospectiva e consecutivamente, e constituíram os Grupos de Estudo e Controle, respectivamente. Oócitos imaturos 146 e 22, respectivamente, dos Grupos Endometriose e Controle) foram submetidos à MIV. Oócitos que apresentaram a extrusão do primeiro corpúsculo polar foram fixados e corados para avaliação dos microtúbulos e cromatina por técnica de imunofluorescência. A análise estatística foi realizada utilizando o teste exato de Fisher, com significância estatística quando $p<0,05$. RESULTADOS: não se observou diferença significativa nas taxas de MIV entre os dois grupos avaliados (45,6 e 54,5\%, respectivamente, nos Grupos Endometriose e Controle). A organização cromossômica e do fuso meiótico foi observada em 18 e 11 oócitos dos Grupos Endometriose e Controle, respectivamente. No Grupo Endometriose, oito oócitos (44,4\%) se apresentavam como metáfase II (MII) normais, três (16,7\%) MII anormais, cinco $(27,8 \%)$ estavam em estágio de telófase I e dois $(11,1 \%)$ sofreram ativação partenogenética. No Grupo Controle, cinco oócitos $(45,4 \%)$ se apresentavam como MII normais, três $(27,3 \%)$ MII anormais, um $(9,1 \%)$ estava em estágio de telófase I e dois $(18,2 \%)$ sofreram ativação partenogenética. Não se observou diferença significativa na porcentagem de anomalias meióticas entre os oócitos em MII dos dois grupos avaliados. CONCLUSÕES: os dados do presente estudo não demonstraram diferença significativa nas taxas de MIV e nas proporções de anomalias meióticas entre os oócitos MIV, provenientes de ciclos estimulados de pacientes com endometriose, quando comparados aos controles. Todavia, sugerem um retardo na conclusão da meiose I nos oócitos provenientes de portadoras de endometriose, pela maior proporção de oócitos em telófase I observada neste grupo.

\section{Abstract}

PURPOSE: to evaluate the meiotic spindle and the chromosome distribution of in vitro mature oocytes from stimulated cycles of infertile women with endometriosis, and with male and/or tubal infertility factors (Control Group), comparing the rates of in vitro maturation (IVM) between the two groups evaluated. METHODS: fourteen patients with endometriosis and eight with male and/or tubal infertility factors, submitted to ovarian stimulation for intracytoplasmatic sperm injection have been prospectively and consecutively selected, and formed a Study and Control Group, respectively. Immature oocytes (46 and 22, respectively, from the Endometriosis and Control Groups) were submitted to IVM. Oocytes presenting extrusion of the first polar corpuscle were fixed and stained for microtubules and chromatin evaluation through immunofluorescence technique. Statistical analysis has been done by the Fisher's exact test, with statistical significance at $\mathrm{p}<0.05$. RESULTS: there was no significant difference in the IVM rates between the two groups evaluated (45.6 and $54.5 \%$ for the Endometriosis and Control Groups, respectively). The chromosome and meiotic spindle organization was

Departamento de Ginecologia e Obstetrícia da Faculdade de Medicina de Ribeirão Preto da Universidade de São Paulo, Laboratório de Ginecologia do Hospital das Clínicas da Faculdade de Medicina de Ribeirão Preto da Universidade de São Paulo, Setor de Reprodução Humana -USP - Ribeirão Preto (SP), Brasil.

'Pós-graduandos do Departamento de Ginecologia e Obstetrícia da Faculdade de Medicina de Ribeirão Preto da Universidade de São Paulo - USP - Ribeirão Preto (SP), Brasil.

2 Bióloga Responsável pelo Laboratório de Reprodução Assistida do Departamento de Ginecologia e Obstetrícia da Faculdade de Medicina de Ribeirão Preto da Universidade de São Paulo -USP - Ribeirão Preto (SP), Brasil.

${ }_{3}^{3}$ Médico Assistente do Setor de Reprodução Humana do Departamento de Ginecologia e Obstetrícia da Faculdade de Medicina de Ribeirão Preto da Universidade de São Paulo -USP - Ribeirão Preto (SP), Brasil.

${ }^{4}$ Professor Titular do Departamento de Ginecologia e Obstetrícia da Faculdade de Medicina de Ribeirão Preto da Universidade de São Paulo -USP - Ribeirão Preto (SP), Brasil.

Doutora, Professora do Departamento de Ginecologia e Obstetrícia da Faculdade de Medicina de Ribeirão Preto da Universidade de São Paulo -USP - Ribeirão Preto (SP), Brasil.

Auxilio à pesquisa: Conselho Nacional de Desenvolvimento Científico e Tecnológico (Edital MCT/CNPq 15/2007 - Universal. Faixa A. Processo 478396/2007-4). 
observed in 18 and 11 oocytes from the Endometriosis and Control Groups, respectively. In the Endometriosis Group, eight oocytes (44.4\%) presented themselves as normal metaphase II (MII), three (16.7\%) as abnormal MII, five (27.8\%) were in telophase stage I and two (1 $1.1 \%)$ underwent parthenogenetic activation. In the Control Group, five oocytes (45.4\%) presented themselves as normal MII, three (27.3\%) as abnormal MII, one (9.1\%) was in telophase stage I and two (18.2\%) underwent parthenogenetic activation. There was no significant difference in meiotic anomaly rate between the oocytes in Mll from both groups. CONCLUSIONS: the present study data did not show significant differences in the IVM or in the meiotic anomalies rate between the IVM oocytes from stimulated cycles of patients with endometriosis, as compared with controls. Nevertheless, they have suggested a delay in the outcome of oocyte meiosis I from patients with endometriosis, shown by the higher proportion of oocytes in telophase I observed in this group.

\section{Introdução}

Vários dados têm apoiado o conceito de diminuição de fecundidade nas portadoras de endometriose ${ }^{1,2}$. Os mecanismos envolvidos na etiopatogênese da infertilidade, em pacientes com endometriose, principalmente nos casos de doença mínima e leve em que não se observa alteração mecânica do trato reprodutivo, ainda não foram bem elucidados.

Novas abordagens para o tratamento da infertilidade relacionada a esta afecção têm surgido, destacando-se a aplicação, cada vez mais rotineira, das técnicas de reprodução assistida de alta complexidade. Encontram-se resultados contraditórios acerca dos resultados da fertilização in vitro em pacientes com endometriose, o que vem sendo foco de diversos estudos e hipóteses nos últimos anos ${ }^{1,3-5}$. Resultados conflitantes de alguns estudos têm sugerido a ocorrência de menores taxas de fertilização, implantação e de gestação em portadoras de endometriose ${ }^{6,7}$, o que poderia ser decorrente do comprometimento da qualidade oocitária e, conseqüentemente, embrionária e/ou de defeitos endometriais ou da interação entre o endométrio e o embrião ${ }^{4,8,9}$. Entretanto, o achado de taxas de implantação semelhantes às do Grupo Controle, em ciclos de doação de oócitos, em pacientes com endometriose, tem sugerido que a qualidade embrionária e não o comprometimento da receptividade endometrial seja a principal responsável pelo comprometimento de implantação verificado neste grupo de pacientes ${ }^{1,10,11}$. A qualidade oocitária, por sua vez, depende da adequada aquisição de maturação citoplasmática e nuclear, sendo a última dependente da presença de um fuso celular normal. O fuso meiótico de oócitos humanos em metáfase II (MII) é uma estrutura temporária e dinâmica, composta por microtúbulos e associada ao córtex oocitário e por sua rede de microfilamentos subcorticais ${ }^{12-14}$, essencial para garantir a fidelidade da segregação cromossômica durante a meiose ${ }^{15-}$ ${ }^{17}$. Desta forma, a fecundação normal e o desenvolvimento embrionário dependem deste controle.

O fuso celular oocitário é extremamente sensível à ação de diversos fatores, entre os quais se podem citar o estresse oxidativo, passível de promover anomalias meióticas, instabilidade cromossômica, indução de apoptose e comprometimento do desenvolvimento embrionário préimplantaçãa ${ }^{18-20}$. Algumas evidências inconclusivas sugerem que o estresse oxidativo, decorrente de um desequilíbrio entre os agentes pró-oxidantes (radicais livres) e os mecanismos antioxidantes de defesa do organismo, possa estar envolvido na etiopatogênese da infertilidade relacionada à endometriose ${ }^{21}$. Apoiando esta hipótese, um estudo recente demonstrou que o fluido peritoneal de mulheres com endometriose promove anomalias nos microtúbulos e cromossomos de oócitos de camundongos, além de induzir aumento da taxa de apoptose embrionária neste modelo animal ${ }^{22}$. Estas anomalias foram reduzidas por meio da suplementação, no meio de cultivo, com L-carnitina, um antioxidante, sugerindo que substâncias presentes no fluido peritoneal de portadoras desta doença promovem comprometimento da qualidade oocitária e embrionária, tendo o estresse oxidativo como provável mediador. Todavia, sabe-se que o fluido folicular contém potente defesa antioxidante $e^{21,23,24}$, e não necessariamente estes resultados, oriundos de estudos in vitro, utilizando modelo animal, podem ser extrapolados para seres humanos. Contudo, caso seja evidenciada uma maior incidência de anomalias do fuso celular e/ou distribuição cromossômica nos oócitos de portadoras de endometriose, submetidas à estimulação ovariana para realização de procedimentos de reprodução assistida, poderia-se justificar ou, ao menos, contribuir para as referidas menores taxas de implantação observadas neste grupo de mulheres, contribuindo também para elucidar um dos mecanismos envolvidos na etiopatogênese da infertilidade relacionada a esta afecção e abrindo perspectivas para novas abordagens terapêuticas. Porém, cabe salientar que não foi encontrado estudo na literatura que tenha avaliado a morfologia do fuso celular e a distribuição cromossômica de oócitos maduros de portadoras de endometriose.

Desta forma, o objetivo deste estudo foi avaliar o fuso meiótico e a distribuição cromossômica de oócitos maduros (maturados in vitro), obtidos de ciclos estimulados de mulheres inférteis com endometriose, comparando com os dados obtidos de oócitos de um Grupo Controle, constituído por casais com fatores masculino e/ou tubário de infertilidade. Como objetivo secundário, comparouse as taxas de maturação oocitária in vitro nos Grupos Endometriose e Controle, de oócitos imaturos, obtidos em ciclos estimulados com gonadotrofinas.

\section{Métodos}

\section{Pacientes e escollha da amostra}

Foram incluídos no presente estudo, consecutivamente, todos os casais submetidos à estimulação ovariana para a 
realização de injeção intracitoplasmática de espermatozóide (ICSI), junto ao Setor de Reprodução Humana do Departamento de Ginecologia e Obstetrícia da Faculdade de Medicina de Ribeirão Preto da Universidade de São Paulo (FMRP-USP), no período de maio de 2006 a junho de 2007. Deste grupo, foram selecionados os que preencheram os critérios de inclusão abaixo descritos e manifestaram o desejo de participar do projeto, mediante a assinatura do termo de consentimento pós-informado.

Foram incluídas pacientes inférteis com idade $\leq 38$ anos, índice de massa corpórea $<30 \mathrm{~kg} / \mathrm{m}^{2}$ e FSH basal $\leq 10 \mathrm{mUI} / \mathrm{mL}$, distribuídas em dois grupos: Grupo Endometriose, constituído por 14 pacientes que preencheram os critérios de inclusão descritos acima, cujo procedimento de reprodução assistida foi indicado pela presença exclusivamente de endometriose, diagnosticada por meio de videolaparoscopia, segundo os critérios definidos pela American Society for Reproductive Medicine $(\text { ASRM })^{25}$, sendo excluídas outras comorbidades. O Grupo Controle foi constituído por oito pacientes que preencheram os critérios acima e cujo procedimento foi indicado devido à presença exclusiva de fator masculino e/ou fator tubário (excluindo-se a presença de hidrossalpinge). Foi considerada como critério de inclusão para ambos os grupos a presença de oócitos imaturos captados e doados que, após maturação in vitro (MIV), tornaram-se maduros (atingiram a metáfase da segunda divisão meiótica, morfologicamente identificada pela presença da extrusão do primeiro corpúsculo polar).

Durante o período de inclusão, foram obtidos, respectivamente, nos Grupos Endometriose (18 ciclos estimulados de 18 pacientes) e Controle (14 ciclos estimulados de 14 pacientes), 46 e 22 oócitos imaturos. Respectivamente, nos Grupos Endometriose e Controle, 21 oócitos (de 14 pacientes) e 12 oócitos (de oito pacientes) apresentaram a extrusão do primeiro corpúsculo polar após a realização da MIV, sendo fixados. A distribuição cromossômica e o fuso meiótico foram observados em 18 e 11 oócitos dos Grupos Endometriose e Controle, respectivamente. Os demais oócitos não foram analisados devido à falha no processo de fixação das estruturas ou perda da célula durante o procedimento (três oócitos no Grupo Endometriose e um no Grupo Controle). As taxas de normalidade e anormalidade do fuso meiótico e distribuição cromossômica foram comparadas entre os grupos.

Foram excluídas do estudo pacientes que utilizaram medicamentos que pudessem interferir na foliculogênese ovariana nos dois meses que antecederam o início da estimulação ovariana (como antiinflamatórios não esteroidais e corticosteróides).

\section{Método}

A estimulação ovariana seguiu o protocolo do setor, que, sucintamente, inclui a programação do início do ciclo mediante uso de contraceptivos orais combinados, dessensibilização hipofisária com agonistas do hormônio liberador de gonadotrofinas ( $\mathrm{GnRH}$, protocolo longo), estimulação ovariana controlada com gonadotrofina (FSH recombinante) e administração de gonadotrofina coriônica humana (hCG), 34 a 36 horas antes da realização da captação oocitária.

As pacientes foram submetidas a uma injeção subcutânea diária de 0,5 mg de acetato de leuprolide (Lupron ${ }^{\circledR}$, Abott, Brasil), iniciado dez dias antes da ultra-sonografia basal, previamente ao início da estimulação ovariana. Foram utilizadas diariamente 200 a 300 unidades de FSH recombinante (Gonal-F ${ }^{\circledR}$, Serono, Brasil e Puregon ${ }^{\circledR}$, Organon, Brasil), nos primeiros seis dias da estimulação ovariana. No sétimo dia da estimulação ovariana foi iniciada a monitorização ultra-sonográfica do ciclo. A partir deste momento, o controle ultra-sonográfico foi realizado diariamente ou a cada dois dias e a dose de gonadotrofinas foi ajustada de acordo com o crescimento folicular observado. A suspensão das gonadotrofinas e do agonista do $\mathrm{GnRH}$ foi realizada quando pelo menos dois folículos apresentavam diâmetro médio de $18 \mathrm{~mm}$, quando foi administrada $250 \mu \mathrm{g}$ de hCG recombinante $\left(\right.$ Ovidrel $^{\circledR}$, Serono, Brasil) às 22:00 horas.

Cerca de 34 a 36 horas após a administração do hCG, a paciente foi submetida à captação oocitária, sob sedação endovenosa com propofol (Diprivan ${ }^{\circledast}$, Astra-Zeneca, Brasil) e citrato de fentanil (Fentanil ${ }^{\circledR}$, Janssen-Cilag, Brasil). A aspiração folicular foi realizada por meio de ultra-sonografia com transdutor transvaginal de $5 \mathrm{MHz}$ acoplado a guia de punção, sob a forma de pool. O material aspirado foi analisado para a identificação e o isolamento dos complexos oócito-cumulus (COC). Após lavagem cuidadosa, os COC identificados foram colocados em placas NUNC (Multidish 4 well Nunclon, Delta SI, Roskilde, Denmark), preenchidas com o meio de cultura Human Tubal Fluid-HEPES (HTF, Irvine Scientific, Santa Ana, CA, USA) suplementado com $10 \%$ Soro Sintético Substituto (SSS, Irvine Scientific), cobertas com óleo mineral (Sigma-Aldrich, Saint Louis, MO, USA), e levados à incubadora, em mistura gasosa de $\mathrm{CO}_{2}$ a $5 \%$, sob temperatura de $37^{\circ} \mathrm{C}$ e $95 \%$ de umidade, por um período de 2 a 4 horas. Após este período, foi realizado o desnudamento oocitário, utilizando-se a exposição dos COC a hialuronidase (H4272 tipo IV-S, Sigma; 80 UI/ $\mathrm{mL}$ ), por 30 segundos, seguida pela remoção mecânica das células do cumulus em meio HTF-SSS, com o auxílio de

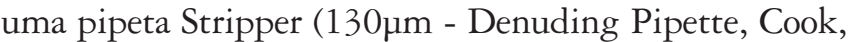
Melbourne, Australia).

Imediatamente após o desnudamento oocitário, realizou-se a análise morfológica para identificação do grau de maturidade dos oócitos, sob visualização ao microscópio de luz. Os oócitos que atingiram a MII, caracterizada morfologicamente pela extrusão do primeiro corpúsculo polar (CP), foram considerados maduros e incubados em gotas de $25 \mu \mathrm{L}$ de $\mathrm{HTF}+\mathrm{SSS}$ a $10 \%$, por uma hora, para, 
então, serem inseminados por meio da realização da ICSI, não sendo, portanto, analisados no presente estudo. Os oócitos que apresentaram sinais de danos mecânicos ou sinais de atresia (citoplasma irregular, escuro ou granulado) foram descartados. Os oócitos que estavam em estágio de vesícula germinativa (VG), isto é, ausência de $\mathrm{CP}$ extruso e presença de VG, ou de metáfase I (MI) (ausência do CP e da VG) foram considerados imaturos e, portanto, sem utilidade clínica para a realização de ICSI, sendo submetidos à MIV, como descrito a seguir, para utilização no presente estudo.

Para MIV, os oócitos imaturos foram transferidos para placas de cultivo em microgotas de $25 \mu \mathrm{L}$ do meio $\mathrm{HTF}+\mathrm{SSS}$ a $10 \%$ (meio previamente equilibrado por período igual ou maior a quatro horas) e levados à incubadora em mistura gasosa de $\mathrm{CO}_{2}$ a $5 \%$, sob condições ideais de temperatura $\left(37^{\circ} \mathrm{C}\right)$ e umidade $(95 \%)$, por período previamente definido. Os oócitos no estágio de VG foram mantidos nestas condições de cultivo por um período de $19 \pm 1$ hora, e aqueles em MI, por $4 \pm 0,5$ hora, conforme curva de maturação previamente realizada neste serviço, utilizando os meios e condições de cultura previamente descritos. Após este período preestabelecido de cultivo, foi realizada novamente a análise do grau de maturação oocitária. Os oócitos que não atingiram a MII após o período descrito assim como os que degeneraram foram descartados. Os oócitos que atingiram o estágio de MII foram fixados para a análise por microscopia de imunofluorescência para caracterização do fuso celular e distribuição cromossômica, conforme previamente descrito $^{26,27}$. A taxa de MIV (definida ao dividir-se o número de oócitos que atinge a MII pelo número total de oócitos imaturos submetidos a MIV) foi calculada para os dois grupos estudados.

Para a fixação e coloração dos oócitos para a microscopia de imunofluorescência, utilizaram-se reagentes adquiridos da Sigma (Sigma Chemical Co., St. Louis (MO), USA), com exceção dos descritos individualmente, a seguir. Os oócitos MIV foram colocados em um fixador adequado para propiciar a estabilidade do fuso celular, composto por um tampão estabilizador $(0,1 \mathrm{M}$ de Pipes, $5 \mathrm{mM}$ de cloreto de magnésio hexahidratado e $2,5 \mathrm{mM}$ de etileno glicol tetra-acético ácido, EGTA, 50\% de óxido de deutério, 0,01\% de apronitina de pulmão bovino, $1 \mathrm{mM}$ de DTT, $1 \mu \mathrm{M}$ de taxol, $0,5 \%$ de Triton-X, $2 \%$ de formaldeído e água de MilliQ), previamente aquecido a $37{ }^{\circ} \mathrm{C}$ por 30 minutos. Os oócitos foram fixados a $37{ }^{\circ} \mathrm{C}$ por 30 minutos e lavados quatro vezes em solução tampão de lavagem (15 minutos cada), composta por $0,02 \%$ de $\mathrm{NaN}_{3}, 0,01 \%$ de Triton-X, 0,2\% de leite desnatado, $2 \%$ de soro normal de cabra, 0,1 M de glicina, 2\% de BSA(V) e PBS, onde ficaram por duas horas a $37^{\circ} \mathrm{C}$. Após esse período, os oócitos foram lavados uma vez na solução tampão de lavagem para incubação com o anticorpo primário (beta-tubulina de camundongo, diluição 1:1.000 em solução de PBS com $0,02 \%$ de $\mathrm{NaN}_{3}$ e $0,1 \%$ de $\mathrm{BSA}(\mathrm{V})$, por quatro horas a $37{ }^{\circ} \mathrm{C}$. Em seguida, foram lavados mais três vezes (15 minutos cada) e incubados com o anticorpo secundário, isto é, imunoglobulina de cabra anti-camundongo FITCconjugada (Zymed Laboratories ${ }^{\circledR}$, Invitrogen Corporation, Calsbad (CA), USA), com diluição 1:200 na mesma solução de diluição acima descrita, por duas horas a $37^{\circ} \mathrm{C}$. Após mais três lavagens de 15 minutos cada, os oócitos foram corados com Hoechst 33342, em meio suporte por dez minutos, à temperatura ambiente, montados entre lâmina e lamínula, estocados a $4^{\circ} \mathrm{C}$ e mantidos no escuro até observação em microscópio de imunofluorescência (Zeiss Axiovert 100TV), realizada dentro de uma semana do preparo da lâmina, para análise morfológica do fuso meiótico e da distribuição cromossômica.

O fuso meiótico foi considerado normal quando apresentava a forma de barril e estava disposto em posição centrípeta em relação ao corpúsculo polar. Os cromossomos apresentaram distribuição normal quando estavam reunidos na placa metafásica e bem alinhados no equador do fuso celular oocitário. Consideraram-se, como em telófase I, os oócitos que apresentavam fuso celular alongado, perpendicular à membrana oocitária, com cromossomos distribuídos nas suas extremidades. Foi considerada como ativação partenogenética a presença de extrusão do segundo corpúsculo polar.

Se os cromossomos se apresentavam dispersos, desalinhados ou fragmentados ou a cromatina feminina se encontrava aglomerada com microtúbulos interfásicos ou o formato do fuso não estava normal ou se o mesmo apresentasse ruptura, este era considerado anormal.

A comparação entre as taxas de MIV e a proporção de anormalidades do fuso celular e distribuição cromossômica entre os dois grupos estudados foi realizada pelo teste exato de Fisher. O grau de significância $(\alpha)$ foi definido em 0,05 (bicaudal), sendo que o valor de $\mathrm{p}<0,05$ foi considerado estatisticamente significativo.

\section{Resultados}

Do total de oócitos imaturos obtidos (68 oócitos), 45,6 (21/46) e 54,5\% (12/22) atingiram o estágio de MII, após MIV nos Grupos Endometriose e Controle, respectivamente. Não se observou diferença significativa entre as taxas de MIV entre os dois grupos avaliados.

A organização cromossômica e do fuso meiótico foi observada em 18 e 11 oócitos dos Grupos Endometriose e Controle, respectivamente. Os demais oócitos não foram analisados devido à falha no processo de fixação das estruturas ou perda da célula durante o procedimento (três oócitos no Grupo Endometriose e um no Grupo Controle). 
Tabela 1. Resultados da análise do fuso celular e configuração cromossômica de oócitos maturados in vitro, obtidos de ciclos estimulados de pacientes com endometriose e infertilidade por fatores masculino e/ou tubário (Grupo Controle)

\begin{tabular}{|c|c|c|c|c|c|c|c|c|c|}
\hline \multirow[t]{2}{*}{ Grupos } & \multirow{2}{*}{$\begin{array}{c}\begin{array}{c}\text { Oócitos } \\
\text { Analisados }\end{array} \\
\mathbf{n}\end{array}$} & \multicolumn{2}{|c|}{$\begin{array}{c}\text { Metáfase II } \\
\text { Normal }\end{array}$} & \multicolumn{2}{|c|}{$\begin{array}{c}\text { Metáfase II } \\
\text { Anormal }\end{array}$} & \multicolumn{2}{|c|}{$\begin{array}{c}\text { Telófase I } \\
\text { Normal }\end{array}$} & \multicolumn{2}{|c|}{$\begin{array}{c}\text { Ativação } \\
\text { Partenogenética }\end{array}$} \\
\hline & & n & $\%$ & n & $\%$ & n & $\%$ & n & $\%$ \\
\hline Endometriose & 18 & 8 & $44,4^{\circ}$ & 3 & $16,7^{b}$ & 5 & $27,8^{c}$ & 2 & 11,1 \\
\hline Controle & 11 & 5 & $45,4^{\circ}$ & 3 & $27,3^{b}$ & 1 & $9,7^{c}$ & 2 & 18,2 \\
\hline
\end{tabular}

Oócitos analisados=oócitos imaturos, obtidos em ciclos estimulados com gonadotrofinas, que após maturação in vitro apresentaram a extrusão do primeiro corpúsculo polar; $n=$ número; mesmos sobrescritos ${ }^{a, b, c}$ dentro da mesma coluna indicam ausência de diferença significativa ( $p>0,05$; teste exato de Fisher).
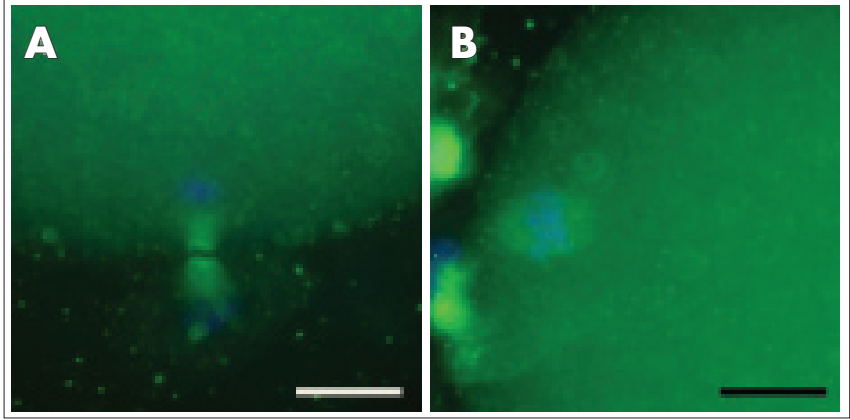

Figura 1- Imagens obtidas por microscopia de fluorescência do fuso celular e dos cromossomos de oócitos humanos, obtidos de ciclos estimulados com gonadotrofinas, submetidos à maturação in vitro; $A=0$ ćcito em telófase | normal; $\mathrm{B}=$ cromossomos adequadamente alinhados na porção mediana de um fuso celular normal de um oócito em metáfase II; verde=microtúbulos constituintes do fuso celular; azul=cromossomos; barra de escala $=10 \mu \mathrm{m}$.

No Grupo Endometriose, dos 18 oócitos analisados, oito $(44,4 \%)$ foram classificados como em MII normal, três $(16,7 \%)$ MII anormal, cinco $(27,8 \%)$ telófase I e dois $(11,1 \%)$ sofreram ativação partenogenética. No Grupo Controle, dos 11 oócitos analisados, cinco $(45,4 \%)$ estavam em MII normal, três $(27,3 \%)$ eram MII anormais, um $(9,1 \%)$ estava em estágio de telófase I e dois $(18,2 \%)$ sofreram ativação partenogenética (Tabela 1 e Figura 1). Não se observou diferença significativa entre a porcentagem de anomalias meióticas nos oócitos em MII dos grupos avaliados (16,7 e 27,3\%, respectivamente, nos Grupos Endometriose e Controle), conforme a Tabela 1.

\section{Discussão}

O presente estudo foi o primeiro na literatura em que foram avaliadas as proporções de anomalias meióticas em oócitos MIV, provenientes de ciclos estimulados de pacientes com endometriose, comparando-as aos de mulheres inférteis, com fatores tubário e/ou masculino (Grupo Controle). Os dados preliminares do presente estudo não evidenciaram diferença significativa nas freqüências de anomalias meióticas entre oócitos em MII dos dois grupos estudados. Por um lado, salienta-se que a pequena casuística apresentada e metodologia utilizada podem comprometer a extrapolação destes dados para oócitos MIV. Por outro, caso os presentes achados sejam confirmados em estudos com maiores casuísticas, estes dados darão subsídios para se afirmar que os danos ao fuso meiótico e à distribuição cromossômica oocitária não são os fatores responsáveis pela sugerida piora da qualidade oocitária em portadoras desta afecção.

Idealmente, para se avaliar a associação entre endometriose e a presença de anomalias meióticas oocitárias, deveria-se proceder à análise do fuso celular e distribuição cromossômica de oócitos maduros (MIV), captados, tanto em ciclos naturais como naqueles estimulados com gonadotrofinas, com a finalidade, inclusive, de se analisar o potencial impacto da estimulação ovariana sobre a gênese de anomalias meióticas oocitárias ${ }^{28-30}$. Todavia, a escassez de oócitos humanos maduros disponíveis e passíveis de serem doados para a realização de pesquisas, utilizando metodologias invasivas, que inviabilizam o uso clínico do referido material, como é o caso da microscopia de imunofluorescência, utilizada no presente estudo, justificou a utilização de oócitos imaturos, obtidos em ciclos estimulados por gonadotrofinas para a realização de ICSI, que atingissem a MII, após a realização de MIV. Por um lado, as baixas proporções de oócitos imaturos obtidos em ciclos estimulados, aliadas à necessidade de que sofressem MIV previamente a sua fixação para imunofluorescência, dificultaram e limitaram a obtenção de oócitos MIV, justificando a pequena casuística apresentada. Por outro, sabe--se que a própria MIV pode cursar com aumento significativo da ocorrência de anomalias meióticas $^{31}$, de tal forma a não se poder extrapolar a proporção de anomalias meióticas encontrada no presente estudo (em oócitos MIV, obtidos de ciclos estimulados) para os oócitos MIV nos ciclos estimulados, ou seja, captados maduros após estimulação ovariana com gonadotrofinas exógenas. Todavia, ressalta-se que a mesma metodologia foi utilizada também no Grupo Controle, de modo que a ocorrência de anomalias meióticas, detectada nos dois grupos, estaria correlacionada a fatores intrínsecos a cada grupo (especialmente a etiologia da infertilidade) e não ao procedimento de MIV per se.

Não se observou diferença significativa nas taxas de MIV entre os dois grupos analisados (taxas de MIV de 45,6 e 54,5\%, respectivamente, nos Grupos Endometriose e Controle), abrindo perspectivas de obtenção de cerca de $50 \%$ de oócitos maduros, a partir da realização de MIV de oócitos imaturos, obtidos em ciclos estimulados. Esta 
estratégia poderia ter utilidade prática, sobretudo nos casos em que forem captados poucos oócitos maduros, e se disporia de oócitos imaturos, que, habitualmente, não são utilizados na prática clínica, possibilitando a obtenção de um maior número de embriões passíveis de serem transferidos a fresco e/ou criopreservados. Estudos futuros serão importantes para analisar a viabilidade e a competência destes oócitos MIV, obtidos em ciclos estimulados, para suportar o desenvolvimento embrionário subseqüente. Ressalta-se a ausência de estudos que tenham comparado às referidas taxas de MIV, utilizando o mesmo meio de maturação empregado no presente estudo. Contudo, Ebner et al. ${ }^{32}$ avaliaram a MIV em meio de cultura BM1 (Biomedical Medium Stage One, NMS Biomedical, Praroman, Suíça), com condições de cultivo similares às empregadas neste estudo, demonstraram taxa de maturação de $44,7 \%$, semelhante aos nossos resultados.

Nesta pesquisa, apenas os oócitos submetidos à MIV que evidenciaram a extrusão do primeiro corpúsculo polar (marcador de maturidade oocitária, segundo critérios de microscopia óptica, rotineiramente utilizado nas clínicas de reprodução assistida) foram fixados e corados para análise por microscopia de imunofluorescência. Um achado interessante foi a tendência a uma maior proporção de oócitos em telófase I no Grupo Endometriose (27,8 e 9,1\% de oócitos em telófase I, respectivamente, nos Grupos Endometriose e Controle), ou seja, oócitos que, apesar de aparentemente maduros, não concluíram a MI. Este achado sugere um potencial retardo ou comprometimento no processo de MI, relacionado à endometriose. Se confirmado, ampliando-se a presente casuística, este dado poderia contribuir para justificar a possível piora da qualidade oocitária relatada em portadoras de infertilidade relacionada à endometriose. Confirmando esta afirmativa, encontrou-se um estudo recente demonstrando que oócitos humanos submetidos à MIV, injetados em telófase I, avaliada por meio da utilização da microscopia de polarização, apresentaram taxas de fertilização significativamente menores que os em $\mathrm{MII}^{33}$. Um outro importante questionamento despertado por este achado é se haveria também entre os oócitos MIV uma porcentagem aumentada de oócitos que, apesar de aparentemente maduros (visualização do primeiro corpúsculo polar), de fato seriam imaturos (em telófase I) nas pacientes com endometriose submetidas a estimulação ovariana para reprodução assistida. Este resultado também poderia ter aplicabilidade clínica, estimulando a avaliação do potencial impacto de postergarmos a inseminação dos oócitos, aparentemente maduros, de mulheres inférteis com endometriose, nos resultados dos procedimentos de reprodução assistida.

Em suma, os dados do presente estudo sugerem que a incidência de anormalidades meióticas dos oócitos que atingem a MII após MIV seja semelhante entre os oócitos MIV, provenientes de ciclos estimulados de mulheres inférteis com endometriose ou fatores tubário e/ou masculino de infertilidade (Grupo Controle). Apesar de as taxas de MIV serem similares entre os dois grupos avaliados, observa-se uma tendência a maior proporção de oócitos em telófase I no Grupo Endometriose, o que poderia ser decorrente de um retardo ou comprometimento da meiose I, neste grupo de pacientes, justificando, pelo menos em parte, a piora sugerida da sua qualidade oocitária. Estudos futuros com maiores casuísticas e, idealmente, avaliando oócitos MIV serão necessários para confirmar os presentes achados. Se confirmados, a análise do impacto do uso da microscopia de polarização, com o objetivo de identificar o estágio real de maturação nuclear oocitária, previamente à realização da ICSI, poderá ser realizada, visando melhorar os resultados dos procedimentos de reprodução assistida.

\section{Agradecimentos}

À contribuição das funcionárias do laboratório de reprodução assistida: Maria Aparecida Carneiro Vasconcelos, Marilda Yamada Dantas, Roberta Cristina Giorgenon, Maria Auxiliadora Pádua Rosa e Sandra Viana, pelo apoio técnico dispensado para a realização do presente artigo.

\section{Referências}

1. Garrido N, Navarro J, Remohí J, Simón C, Pellicer A. Follicular hormonal environment and embryo quality in women with endometriosis. Hum Reprod Update. 2000;6(1):67-74.

2. Garrido N, Navarro J, García-Velasco J, Remoh J, Pellice A, Simón C. The endometrium versus embryonic quality in endometriosisrelated infertility. Hum Reprod Update. 2002;8(1):95-103.

3. Garcia-Velasco JA, Arici A. Is the endometrium or oocyte/embryo affected in endometriosis? Hum Reprod. 1999;14 Suppl 2: 77-89.
4. Kumbak B, Kahraman S, Karlikaya G, Lacin S, Guney A. In vitro fertilization in normoresponder patients with endometriomas: comparison with basal simple ovarian cysts. Gynecol Obstet Invest. 2008;65(3):212-6.

5. Fernando S, Breheny S, Jaques AM, Halliday JL, Baker G, Healy D. Preterm birth, ovarian endometriomata, and assisted reproduction technologies. Fertil Steril. In press 2008.

6. Barnhart K, Dunsmoor-Su R, Coutifaris C. Effect of endometriosis on in vitro fertilization. Fertil Steril. 2002;77(6): 1 148-55. 
7. Al-Fadhli R, Kelly SM, Tulandi T, Tanr SL. Effects of different stages of endometriosis on the outcome of in vitro fertilization. J Obstet Gynaecol Can. 2006;28(10):888-91.

8. Brizek CL, Schlaff S, Pellegrini VA, Frank JB, Worrilow KC. Increased incidence of aberrant morphological phenotypes in human embryogenesis-an association with endometriosis. J Assist Reprod Genet. 1995; 12(2): 106-12.

9. Pellicer A, Oliveira N, Ruiz A, Remohí J, Simón C. Exploring the mechanism(s) of endometriosis-related infertility: an analysis of embryo development and implantation in assisted reproduction. Hum Reprod. 1995; 10 Suppl 2:91-7.

10. Pellicer A, Navarro J, Bosch E, Garrido N, Garcia-Velasco JA, Remohí J, et al. Endometrial quality in infertile women with endometriosis. Ann N Y Acad Sci. 2001 ;943:122-30.

11. Katsoff B, Check JH, Davies E, Wilson C. Evaluation of the effect of endometriosis on oocyte quality and endometrial environment by comparison of donor and recipient outcomes following embryo transfer in a shared oocyte program. Clin Exp Obstet Gynecol. 2006;33(4):201-2.

12. Kim NH, Chung HM, Cha KY, Chung KS. Microtubule and microfilament organization in maturing human oocytes. Hum Reprod. 1998;13(8):2217-22.

13. Wang $W H$, Keefe DL. Prediction of chromosome misalignment among in vitro matured human oocytes by spindle imaging with the PolScope. Fertil Steril. 2002;78(5):1077-81.

14. Mandelbaum J, Anastasiou $O$, Lévy R, Guérin JF, de Larouzière $V$, Antoine JM. Effects of cryopreservation on the meiotic spindle of human oocytes. Eur J Obstet Gynecol Reprod Biol. 2004;113 Suppl 1:S17-23.

15. De Santis L, Cino I, Rabellotti E, Calzi F, Persico P, Borini A, et al. Polar body morphology and spindle imaging as predictors of oocyte quality. Reprod Biomed Online. 2005; 11 (1):36-42.

16. Volarcik K, Sheean L, Goldfarb J, Woods L, Abdul-Karim FW, Hunt $P$. The meiotic competence of in-vitro matured human oocytes is influenced by donor age: evidence that folliculogenesis is compromised in the reproductively aged ovary. Hum Reprod. 1998; 13(1): 154-60.

17. Van Blerkom J, Davis P. Differential effects of repeated ovarian stimulation on cytoplasmic and spindle organization in metaphase II mouse oocytes matured in vivo and in vitro. Hum Reprod. $2001 ; 16(4): 757-64$.

18. Liu L, Trimarchi JR, Navarro P, Blasco MA, Keefe DL. Oxidative stress contributes to arsenic-induced telomere attrition, chromosome instability, and apoptosis. J Biol Chem. 2003;278(34): 31998-2004.

19. Navarro PA, Liu L, Keefe DL. In vivo effects of arsenite on meiosis, preimplantation development, and apoptosis in the mouse. Biol Reprod. 2004;70(4):980-5.

20. Navarro PA, Liu L, Ferriani RA, Keefe DL. Arsenite induces aberrations in meiosis that can be prevented by coadministration of N-acetylcysteine in mice. Fertil Steril. 2006;85 Suppl 1: $1187-94$.

21. Campos Petean C, Ferriani RA, Dos Reis RM, Dias de Moura M, Jordão AA Jr, Andrea de Albuquerque Salles Navarro P. Lipid peroxidation and vitamin $E$ in serum and follicular fluid of infertile women with peritoneal endometriosis submitted to controlled ovarian hyperstimulation: a pilot study. Fertil Steril. Feb 2. [Epub ahead of print]

22. Mansour G, Abdelrazik H, Sharma RK, Radwan E, Falcone T, Agarwal A. L-carnitine supplementation reduces oocyte cytoskeleton damage and embryo apoptosis induced by incubation in peritoneal fluid from patients with endometriosis. Fertil Steril. In press 2008.

23. Jozwik M, Wolczynski S, Jozwik M, Szamatowicz M. Oxidative stress markers in preovulatory follicular fluid in humans. Mol Hum Reprod. 1999;5(5):409-13.

24. Carbone MC, Tatone C, Delle Monache S, Marci R, Caserta $D$, Colonna $R$, et al. Antioxidant enzymatic defences in human follicular fluid: characterization and age-dependent changes. Mol Hum Reprod. 2003;9(1 1):639-43.

25. Revised American Society for Reproductive Medicine classification of endometriosis: 1996. Fertil Steril. 1997;67(5):817-21.

26. Allworth $A E$, Albertini DF. Meiotic maturation in cultured bovine oocytes is accompanied by remodeling of the cumulus cell cytoskeleton. Dev Biol. 1993;158(1):101-12.

27. Liu L, Keefe DL. Ageing-associated aberration in meiosis of oocytes from senescence-accelerated mice. Hum Reprod. 2002;17(10): 2678-85.

28. Lee ST, Han HJ, Oh SJ, Lee EJ, Han JY, Lim JM. Influence of ovarian hyperstimulation and ovulation induction on the cytoskeletal dynamics and developmental competence of oocytes. Mol Reprod Dev. 2006;73(8): 1022-33.

29. Baart EB, Martini E, Eijkemans M, Van Opstal D, Beckers NG, Verhoeff $A$, et al. Milder ovarian stimulation for in-vitro fertilization reduces aneuploidy in the human preimplantation embryo: a randomized controlled trial. Hum Reprod. 2007;22(4):980-8.

30. Grupen CG, Gilchrist RB, Nayudu PL, Barry MF, Schulz SJ, Ritter L, et al. Effects of ovarian stimulation, with and without human chorionic gonadotrophin, on oocyte meiotic and developmental competence in the marmoset monkey (Callithrix jacchus). Theriogenology. 2007;68(6):861-72

31. Li Y, Feng HL, Cao YJ, Zheng GJ, Yang Y, Mullen S, et al. Confocal microscopic analysis of the spindle and chromosome configurations of human oocytes matured in vitro. Fertil Steril. 2006;85(4):827-32.

32. Ebner T, Moser M, Sommergruber M, Shebl O, Tews G. Incomplete denudation of oocytes prior to ICSI enhances embryo quality and blastocyst development. Hum Reprod. 2006;21 (1 1):2972-7.

33. Hyun CS, Cha JH, Son WY, Yoon SH, Kim KA, Lim JH. Optimal ICSI timing after the first polar body extrusion in in vitro \matured human oocytes. Hum Reprod. 2007;22(7):1991-5. 